\title{
Effets de la farine de chenille (Imbrasia oyemensis) sur les performances de croissances et le rendement des organes de poulets de chair en Côte d'Ivoire
}

\author{
Massé DIOMANDE ${ }^{1, *}$, Maxwell Grat Avit BEUGRE ${ }^{1}$, Benjamin Kan KOUAME ${ }^{1}$ et \\ Louis Guichard BOHOUA ${ }^{2}$ \\ ${ }^{I}$ Departement de Biochimie et Microbiologie, UFR Agroforesterie, \\ Université Jean Lorougnon Guédé, Côte d'Ivoire. \\ ${ }^{2}$ UFR Sciences et Technologie des Aliments, Université Nangui Abrogoua, Côte d'Ivoire. \\ *Auteur correspondant ; E-mail: masse635@gmail.com; Tel: (00225)05702160/(00225)08665573
}

\section{RESUME}

L'objectif de cette étude était d'évaluer l'influence de la farine de chenille (Imbrasia oyemensis) sur les performances de croissances et le rendement des organes de poulets de chair en Côte d' Ivoire. Ainsi, la farine de chenille (Imbrasia oyemensis) a été incorporée aux rations alimentaires des poulets comme substitut de farine de poisson (Thunnus albacares). Le taux de substitution a été de $0 ; 1 / 3 ; 2 / 3$ et 3/3 respectivement dans les rations R0 ; R33 ; R66 et R100. Quatre lots de 25 poulets (Cobb 500) de 2 semaines d'âge ont été nourris avec ces quatre rations durant 5 semaines d'expérimentation. Les résultats de l'analyse chimique des deux farines ont montré que celle de chenille est plus riche en protéines $(53,57 \%)$ que celle de poisson $(38,56 \%)$. Elle a été plus pauvre en cendres $(2,17 \%)$ que la farine de poisson $(7,73 \%)$. L'évaluation des performances zootechniques des poulets nourris aux 4 rations a montré des variations non significatives des poids vifs (1803$2241 \mathrm{~g})$, gains moyens quotidiens $(41,62-45,99 \mathrm{~g} / \mathrm{sujet})$, consommations alimentaires individuelles $(72,92-$ $87,10 \mathrm{~g} / \mathrm{sujet} / \mathrm{j})$, indices de consommation $(1,88-2,19)$ selon les taux variables de farine de chenilles dans les rations des sujets. Il en a été de même que les rendements carcasses $(78,79-92,62 \%)$, gras abdominaux $(1,44-$ $2,11 \%)$ cœur (0,39-0,45\%), foie (2,09-3,09\%), gésier (1,93-2,60\%). La mortalité n'a pas été corrélée avec les teneurs de farine de chenille des régimes alimentaires des poulets. Ainsi, la chenille (Imbrasia oyemensis) peut être valablement incorporée dans l'alimentation des poulets de chair sans risque de modifier la production et les caractéristiques des viandes. Elle peut être recommandée dès lors aux aviculteurs comme ressource alternative de protéines pour la volaille dans les pays tropicaux.

(C) 2018 International Formulae Group. All rights reserved.

Mots clés: Chenille, Imbrasia oyemensis, ration alimentaire, performances zootechniques, poulet de chair.

\section{Effects of caterpillar meal (Imbrasia oyemensis) on growth performance and yield of broiler organ in Côte d'Ivoire}

\begin{abstract}
The aim of this study was to evaluate the influence of caterpillar meal (Imbrasia oyemensis) on the growth performance and yield of broiler organs in Côte d'Ivoire. This caterpillar meal (Imbrasia oyemensis) has been incorporated into chicken feed as a substitute for fish meal (Thunnus albacares). The substitution rate was
\end{abstract}


0; 1/3; 2/3 and 3/3 respectively in rations R0; R33; R66 and R100. Four batches of 25 chickens (Cobb 500) of 2 weeks of age were fed with these four rations during 5 weeks of experimentation. The results of the chemical analysis of the two flours showed that the caterpillar flour is richer in protein $(53.57 \%)$ than that of fish $(38.56 \%)$. It was lower in ash $(2.17 \%)$ than fishmeal $(7.73 \%)$. The evaluation of the zootechnical performances of the chickens fed to the 4 rations showed nonsignificant variations of the live weight (1803-2241 g), average daily earnings (41.62-45.99 g / subject), individual food consumption (72.92-87.10 g/ subject / j), consumption indices (1.88-2.19) according to the variable rates of caterpillar meal in the rations of the subjects. It was the same as carcass yields (78.79-92.62\%), abdominal fat (1.44-2.11\%) heart (0.39-0.45\%), liver (2.09-3.09\%), gizzard (1.93-2.60\%). Mortality was not correlated with caterpillar meal levels in chicken diets. Thus the caterpillar (Imbrasia oyemensis) can be validly incorporated into the diet of broilers without the risk of modifying the production and characteristics of the meat. It may, therefore, be recommended to poultry farmers as an alternative protein resource for poultry in tropical countries.

(C) 2018 International Formulae Group. All rights reserved.

Keywords: Caterpillar, Imbrasia oyemensis, feed ration, zootechnical performance, broiler.

\section{INTRODUCTION}

La Côte d'Ivoire s'est lancée dans l'aviculture industrielle depuis une dizaine d'année (Pousga et al., 2005). Ainsi, de nombreux éleveurs s'adonnent à l'aviculture autour des grandes métropoles comme Abidjan. Malgré cela, la production nationale n'arrive pas à couvrir la demande croissante. D'où les importations de viande de volaille en Côte d'Ivoire qui, de 2000 tonnes en 2000 sont passées à 14000 tonnes en 2004 (Essoh, 2006). La problématique de l'approvisionnement en intrants alimentaires est de nos jours d'autant plus cruciaux qu'on assiste sur le marché international au renchérissement du coût des matières premières protéiques, notamment la farine de poisson, le soja et l'arachide (Doumbia, 2002). Dans ces conditions, la recherche et la valorisation de ressources alimentaires alternatives et disponibles localement dans l'alimentation des poulets devraient permettre d'améliorer leur productivité (Soniaya et Gueye, 1998). Parmi ces ressources alternatives figurent, en bonne place, les insectes comestibles dont Imbrasia oyemensis. En effet, ces chenilles sont disponibles et facile d'accès en zone forestière et peuvent constituer, de par leurs qualités nutritives, des sources de protéines et d'énergie (Malaisse et Lognay, 2004). En Côte d'Ivoire, où existent plusieurs variétés d'insectes comestibles, aucune étude n'a été consacrée à l'incorporation de farine de chenille dans la ration des poulets de chair, d'où l'intérêt du présent travail.

L'objectif général était la recherche de ressources alternatives de protéines pour la volaille par la valorisation de la farine de chenille (Imbrasia oyemensis) dans les régimes alimentaires de poulets de chair. De façon spécifique, il s'agit d'évaluer les effets de l'incorporation de la farine de chenille (Imbrasia oyemensis) dans le régime sur les performances de croissance, les rendements de la carcasse, des organes et du gras abdominal des poulets de chair en Côte d' Ivoire.

\section{MATERIEL ET METHODES Matériel biologique}

Le matériel biologique est constitué de farines de chenille (Imbrasia oyemensis) et de poisson (Thunnus albacares) d'une part, et d'autres part, de poulet de chair, souche cobb500 (Figures 1 et 2).

\section{Formulations des rations}

Quatre (4) rations expérimentales de type démarrage croissance pour poulets de chair ont été formulées. Il s'agit des rations $\left(\mathrm{R}_{0}, \mathrm{R}_{33}, \mathrm{R}_{66}\right.$ et $\left.\mathrm{R}_{100}\right)$ d'aliments Ivograin à différents ingrédients (maïs, son de blé, tourteaux de coton et de soja, sel, vitamines, phosphate bi calcique ont été maintenues constantes). Cet aliment a été complété par la farine de chenille (Imbrasia oyemensis) et de poisson incorporée respectivement à $0 \%$, 
$33 \%, 66 \%, 100 \%$ comme substitut de la farine de poisson (Thunnus albacares) habituellement utilisée comme source principale de protéines dans l'alimentation de poulet de chair. La préparation consistait à mélanger manuellement les quantités des différentes matières premières choisies. Dans chaque sac d'aliment Ivograin, $2 \mathrm{~kg}$ de farine de chenille et de poisson dans les proportion alternative de $0 \%, 33 \%, 66 \%, 100 \%$ ont été mélangés de façon à avoir une ration bien homogène.

\section{Analyse physico-chimique des farines et rations de poulet}

$\mathrm{Au}$ laboratoire, les chenilles sèches achetées au grand marché de Man ont été broyées au mixeur. Les taux d'humidité et de cendres de la farine de chenille séchée et de poisson ont été déterminés selon la méthode AOAC (1995). Les protéines brutes ont été dosées selon la méthode de BIPEA (1976) utilisant le Kjeldhal. La matière grasse à été extraite selon la méthode AOAC (1995) utilisant le soxhlet. Les glucides ont été dosés selon la méthode de Bertrand et Thomas (1910). La valeur énergétique correspondant à l'énergie disponible est calculée à l'aide des coefficients spécifiques d'Atwater et Benedict (1902) pour les protéines, les lipides et les glucides.

\section{Evaluations des performances zootechniques des poulets}

Quatre (4) lots de 25 poulets de chair expérimentaux sans répétition ont été nourrit aux différentes rations afin de faire une évaluation des performances zootechniques des poulets de chair (poids vif, gain moyen quotidien, consommation alimentaire individuelle, indice de consommation, Rendements carcasse, gras abdominal, organe et taux de mortalité). Depuis la phase de démarrage, les animaux ont été pesés individuellement et répartis de manière aléatoire en fonction du traitement subi, la densité étant de 10 sujets $/ \mathrm{m}^{2}$ jusqu'à l'abattage des animaux. Les sujets ont subi le traitement sanitaire classique. Ensuite, chaque groupe de poulets a été nourri avec de l'aliment expérimental jusqu'à la vente. Les quantités d'aliment servies et les refus ont été pesés pour en déduire la consommation.

Poids vifs des poulets de chair (PV)

Les poulets ont été pesés chaque semaine afin de déterminer le poids vif moyen. Il est le rapport de la somme des poids des individus d'un même lot par leur effectif:

$$
\mathrm{PV}=\left(\sum \mathrm{PVi} / \mathrm{EP}\right) \text { avec } \sum \mathrm{PVi}:
$$
somme des masses des sujets; EP: effectif des poulets

\section{Gains moyens quotidiens des poulets pendant la période d'essai (GMQ)}

Les mesures hebdomadaires des poids répertoriés, ont permis de calculer le gain moyen quotidien en faisant le rapport du gain pondéral pendant une période sur la durée correspondante: $\mathrm{GMQ}=(\mathrm{G} \mathrm{P} / \mathrm{d} \mathrm{p}) \times 100$ avec $G$ P: Gain de poids ( $g$ ) pendant une période ; DP : Durée de la période (jours).

\section{Consommations alimentaires individuelles}

\section{(CAI)}

La consommation alimentaire individuelle permet d'évaluer les quantités d'aliments consommés par animal sur une période de temps déterminée. Elle se calcule à partir de la quantité d'aliment distribuée et celle refusée. $\mathrm{CAI}=(\mathrm{QAD}-\mathrm{QAR}) / \mathrm{N} \mathrm{S}$ avec QAD : Quantité d'aliments distribuée (g) par jour QAR: Quantité d'aliments refusée $(\mathrm{g})$ par jour ; N S : Nombre de sujets

\section{Indice de consommation (IC)}

C'est le rapport entre la quantité moyenne d'aliment consommée sur une période donnée et le gain de poids moyen correspondant à cette période.

$\mathrm{IC}=\mathrm{QAC} / \mathrm{GP}$ avec QAC : quantité d'aliments consommée pendant une période (g) ; GP : Gain de poids durant la période (g)

\section{Rendements organes (RO)}

Il consiste à faire le rapport entre le poids de l'organe (foie, cœur, gésier) et le poids vif du sujet à l'abattage. Il est exprimé en pourcentage : $\mathrm{R} \mathrm{O}=\left(\mathrm{M} \mathrm{O} / \mathrm{M} \mathrm{P}_{\mathrm{V}}\right) \times 100$ avec MO : Masse organe; $M P_{\mathrm{v}}$ : Masse du poulet vif.

\section{Rendement carcasse (RC)}

Le rendement carcasse (\%) est calculé en faisant le rapport du poids carcasse sur le 
poids vif du sujet à l'abattage exprimé en pourcentage. Après 49 jours d'expérimentation, 12 poulets de chair ont été abattus, plumés et pesés. Ensuite les têtes, pattes et viscères des poulets ont été enlevés afin d'obtenir les carcasses qui ont été pesées. Ainsi, les rendements carcasse ont été calculés selon la formule ci-dessous : $\quad \mathrm{RC}=(\mathrm{M} \mathrm{C} /$ $\mathrm{MP}_{\mathrm{p}}$ ) $\mathrm{x} 100$ avec

$\mathrm{MC}$ : Masse de carcasse ; $\mathrm{M} \mathrm{P}_{\mathrm{p}}$ : Masse du poulet plumé.

\section{Taux de gras abdominal (TGA)}

Pour déterminer le taux de gras abdominal, la graisse abdominale des poulets a été extraite et pesée. Le taux de gras abdominal a été calculé selon la formule suivante $:$ TGA $=\left(\right.$ MGA $\left./ \mathrm{MP}_{\mathrm{p}}\right) \times 100 \mathrm{avec}$

MGA: Masse de la graisse abdominale ; $\mathrm{MP}_{\mathrm{p}}$ : Masse du poulet plumé.

\section{Taux de mortalité (TM)}

Le taux de mortalité (\%) correspond au rapport du nombre total de poulets morts sur l'effectif initial des sujets: $\mathrm{TM}=(\mathrm{NM} / \mathrm{E} \mathrm{P})$ x 100 avec NM : Nombre de poulets morts au cours d'une période ; EP : Effectif en début de période.

\section{Analyse statistique des données}

Les données recueillies à l'issue de la caractérisation physicochimique des échantillons ont été soumises à des analyses statistiques. Ainsi, une analyse de variance multidimensionnelle a été réalisée aux fins d'apprécier l'existence de différence entre les échantillons étudiés. Des tests de comparaison multiples (Turkey HSD) ont été conduits lorsque la différence a été révélée comme significative $(p<0,05)$ aux fins de séparer les différents échantillons. Pour ces traitements statistiques, le logiciel STATISTICA 7.0 a été utilisé.

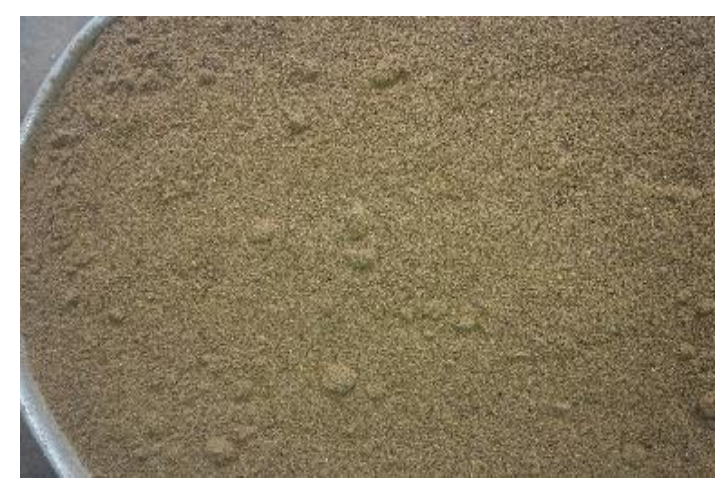

Figure 1 : Farine de chenille (Imbrasia oyemensis).

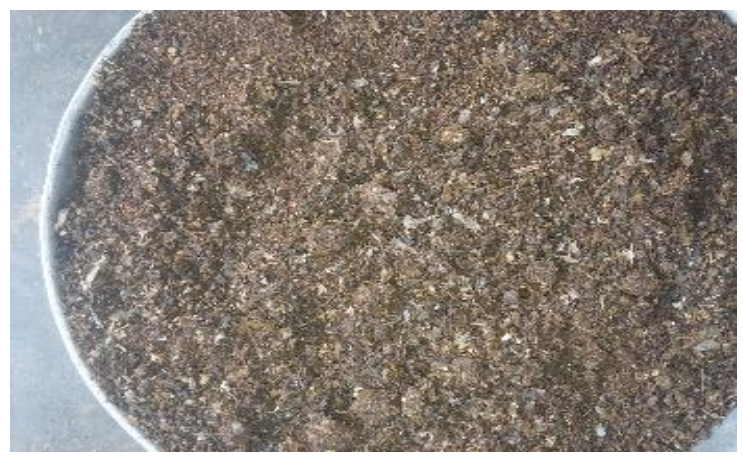

Figure 2 : Farine de poisson (Thunnus albacares). 


\section{RESULTATS}

Composition chimique des farines de chenille (Imbrasia oyemensis) et de poisson (Thunnus albacares)

La farine de chenille est plus riche $(53,57 \%)$ en protéine que celle de poisson $(38,56 \%)$. En revanche, elle est plus pauvre en cendres $(2,17 \%$ contre $7,73 \%)$ que celle de poisson. Les autres composantes chimiques des ces deux farines sont similaires (Tableau $1)$.

\section{Composition physicochimique des rations Ration de démarrage}

Le taux d'humidité est identique dans les rations R0 à R100. Le taux de cendre diminue à mesure que le taux de chenille augmente dans les rations. La teneur en glucide est presque constante de la R0 à R100. La valeur énergétique de la ration R100 est plus élevée que celle des autres rations qui sont sensiblement identiques. Le taux de protéine augmente progressivement de R0 à R100 (Tableau 2).

\section{Ration de croissance}

Le taux d'humidité est constant dans les rations. Le taux de cendre et de matière grasse diminue avec l'augmentation du taux de chenille dans les rations. Quant à la teneur en protéine, elle est faible dans la ration R0 par rapport aux autres rations ou elle a une teneur constante. La teneur en glucides est faible dans la ration R33 et est plus élevés dans les autres rations. La valeur énergétique diminue lorsque la quantité de chenille augmente (Tableau 3).

\section{Performances zootechniques des poulets Poids vifs (PV)}

Une augmentation pondérale est constatée dans tous les traitements en fonction du temps. Pendant tout l'essai, les poids des oiseaux ne diffèrent pas significativement $(p$ $>0,05$. A la fin de l'expérience, le poids des oiseaux du traitement R0 est de 2241,60 g. Quant aux traitements R33, R66 et R100, les poids sont respectivement de $1785,30 \mathrm{~g}$, de $1908,60 \mathrm{~g}$ et de 1803,20 g. Les oiseaux ayant reçu la farine de chenille en complément (R100) ont les plus faibles poids alors que les poids les plus élevés ont été enregistré chez les animaux qui ont consommé l'aliment complémentaire de farine de poisson (R0). Cependant, l'analyse statistique n'a montré aucune différence significative entre ces poids (Figure 3).

\section{Gain moyen quotidien (GMQ)}

Pendant le démarrage, le GMQ des oiseaux du traitement R0 $(42,98 \mathrm{~g})$ est supérieur respectivement aux GMQ des traitements R100 (40,26 g), R66 (39,26 g) et R33 (34,59 g). Pendant la croissance, le GMQ des oiseaux du traitement R0 $(56,48 \%)$ est supérieur respectivement aux GMQ des traitements R66 (56,14 g), R33 (52,33 g) et R100 (47,34 g). Le plus faible GMQ a été enregistré au niveau du traitement R33. Sur l'ensemble de l'essai, les GMQ des oiseaux des traitements sont, R0 (52,38 g), R33 (41,69 $\mathrm{g})$ et R66 (45,99 g) et R100 (43,09 g). Les oiseaux du traitement R0 ont donc affiché le meilleur GMQ (52,38 g), suivi du traitement R66 (45,99 g) et enfin le traitement R100 $(43,09 \mathrm{~g})$. Il faut cependant indiquer qu'aucune différence significative n'est constatée entre les GMQ des poulets soumis aux différents traitements $(p>0,05)$ (Tableau 4).

\section{Consommations alimentaires individuelles (CAI)}

Les évolutions de la consommation alimentaire des poulets de chair sont présentées dans le Tableau 5. Au démarrage, la consommation R0 (81,16 g/sujet/j) est supérieure à celle des autres R33 (74,48 g/sujet/j, R66 (80,36 g/sujet/j), R100 (79,92 $\mathrm{g} / \mathrm{sujet} / \mathrm{j})$. Pendant la croissance les consommations R0 et R100 (86,00 g/sujet/j) sont identiques et supérieures à celles de R33 $(70,59 \mathrm{~g} / \mathrm{sujet} / \mathrm{j})$ et R66 (80,00 g/sujet/j). Pendant tout l'essai les consommations alimentaires des animaux des traitements R33 (72,92 g / sujet / j) et R66 (80,22 g/sujet/j) sont restées inférieures à celles des autres traitements R0 $(87,10 \mathrm{~g} / \mathrm{sujet} / \mathrm{j})$ et R100 $(86,35 \mathrm{~g} / \mathrm{sujet} / \mathrm{j}) \quad$ les consommations alimentaires individuelles les plus élevées sont notées pour R0 au démarrage, en croissance et durant tout l'essai. En revanche les 
consommations alimentaires individuelles les plus faibles sont enregistrées pour la ration R33. Pendant tout l'essai, aucune différence significative n'est enregistrée entre les différents traitements $(\mathrm{p}>0,05)$ (Tableau 5).

\section{Indice de consommation (IC)}

Pendant tout l'essai les IC des oiseaux ne diffèrent pas significativement. Pendant tout l'essai, les poids des oiseaux n'ont pas différé pas significativement. Les indices de consommation (IC) sont plus élevés au démarrage $(2,17$ à 2,32$)$ et plus faibles en période de croissance $(1,35$ à 2,09). Les indices de consommation varient entre 1,88 et 2,19 pendant toute la période d'essai. Les faibles valeurs sont enregistrées pour les rations R66 au démarrage $(2,17)$, R33 en croissance $(1,35)$ et R66 $(1,88)$ durant l'essai. En revanche les valeurs les plus élevées sont notées pour les rations R0 démarrage, R100 en croissance et R100 pour toute la période de l'essai (Tableau 6).

\section{Rendements carcasse et gras abdominal}

Les rendements de carcasse ont varié de $78,79 \%$ à 92,62\%. La plus grande valeur a enregistré pour R33 $(92,62 \%)$ et la plus petite pour R66 $(78,79 \%)$. Les rendements du gras abdominal ont varié entre $1,44 \%$ et $2,11 \%$ avec la plus grande valeur pour R66 $(2,11 \%)$ et la plus faible R0 $(1,44 \%)$. Toutefois, il $\mathrm{n}$ y a pas de différence significative entre les carcasses et les gras abdominaux des différents traitements (Tableau 7).

\section{Rendements autres organes}

Les rendements du cœur ont varié de 0,3 à 0,4. La plus grande valeur a été enregistrée pour R0, R33 et R66 $(0,4)$ et la plus petite pour R100 $(0,3)$. Les rendements du foie se situaient entre $2 \%$ et $3 \%$ avec la plus grande valeur pour R33 (3\%) et la plus faible R0 (2\%). Les rendements du gésier variaient entre 1,9 et $2,6 \%$ (R100). Les rations R33 et R66 ayant présenté respectivement 2,5 et $2,1 \%$ (Tableau 7).

\section{Taux de mortalité (TM)}

Aucun mort n'a été enregistré dans les lots R0 et R100. En revanche, le taux de mortalité le plus élevé a été noté pour le lot R66 (40\%) suivi de R33 (32\%) (Tableau 8).

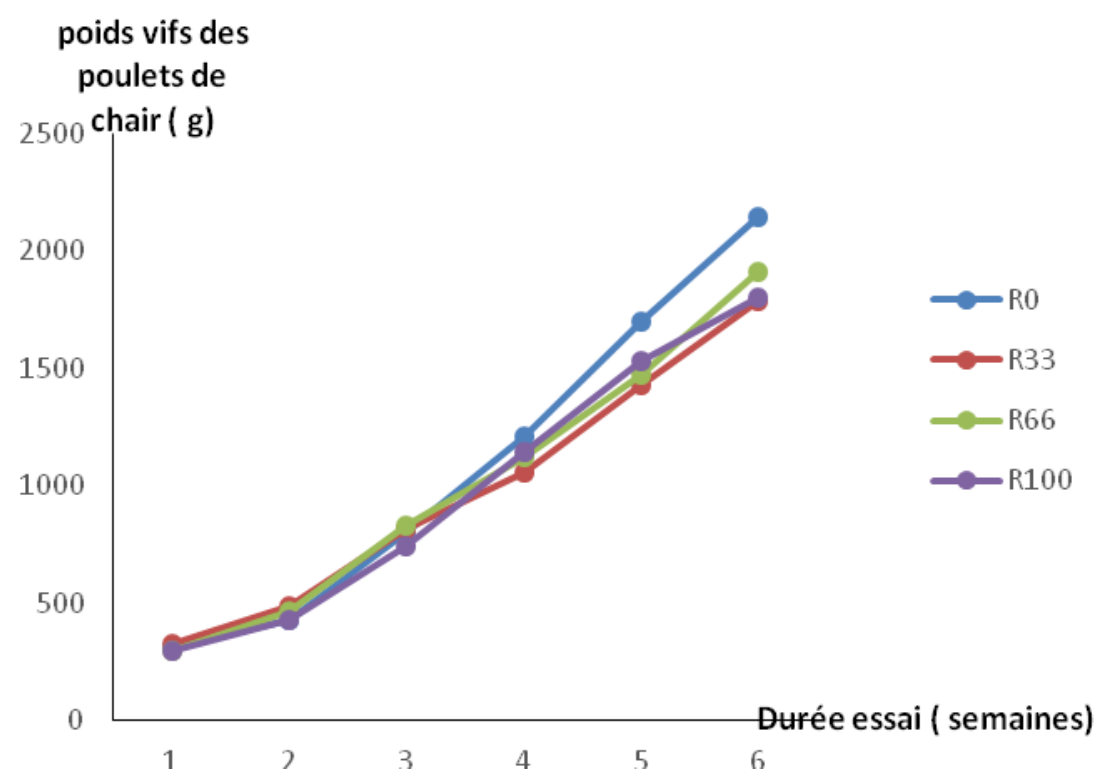

Figure 3: Evolution des poids vifs (PV) des poulets au cours de l'essai. 
Tableau 1: Composition chimique des farines de chenille et poisson.

\begin{tabular}{lcccc}
\hline & \multicolumn{3}{c}{ Composition chimique (\% MS) } \\
\hline & Matière Sèche & Humidité & Cendre & Matières Grasses \\
\hline $\mathrm{Fp}$ & $95,3 \pm 0,32 \mathrm{a}$ & $4,70 \pm 0,11 \mathrm{~b}$ & $7,73 \pm 0,09 \mathrm{a}$ & $20,78 \pm 0,24 \mathrm{c}$ \\
$\mathrm{Fc}$ & $94,43 \pm 0,33 \mathrm{a}$ & $5,56 \pm 0,33 \mathrm{~b}$ & $2,17 \pm 1,41 \mathrm{~b}$ & $38,56 \pm 0,14 \mathrm{a}$ \\
\hline
\end{tabular}

Les valeurs moyennes de la même colonne indexée de la même lettre ne sont pas statistiquement différents selon le test de Turkey au seuil $5 \%$. Fc : farine de poisson, Fp : farine de chenille.

Tableau 2: Ration de démarrage.

Composition chimique (\% MS)

\begin{tabular}{|c|c|c|c|c|c|c|c|}
\hline & Matière Sèche & Humidité & Cendre & Matières Grasses & Protéines & Glucides & VE (Kcal/100g) \\
\hline R0 & $93,88 \pm 0,01 \mathrm{a}$ & $6,12 \pm 0,01 \mathrm{a}$ & $7,50 \pm 0,11 \mathrm{a}$ & $7,91 \pm 0,09 \mathrm{a}$ & $21,39 \pm 0,30 \mathrm{a}$ & $57,08 \pm 0,39 a$ & $385,07 \pm 0,42 \mathrm{a}$ \\
\hline R33 & $93,25 \pm 0,33 a$ & $6,75 \pm 0,33 a$ & $7,12 \pm 0,08 \mathrm{a}$ & $7,33 \pm 0,10 \mathrm{a}$ & $22,25 \pm 0,14 b$ & $56,54 \pm 0,66 a$ & $381,17 \pm 1,18 \mathrm{a}$ \\
\hline R66 & $93,98 \pm 0,03 a$ & $6,02 \pm 0,03 \mathrm{a}$ & $6,42 \pm 0,04 b$ & $7,24 \pm 0,31 \mathrm{a}$ & $22,67 \pm 0,07 b$ & $57,65 \pm 0,36 a$ & $386,17 \pm 1,69 a$ \\
\hline $\mathrm{R} 100$ & $93,94 \pm 0,02 \mathrm{a}$ & $6,06 \pm 0,02 \mathrm{a}$ & $6,17 \pm 0,04 b$ & $8,84 \pm 0,14 b$ & $23,74 \pm 0,07 \mathrm{c}$ & $55,17 \pm 0,14 b$ & $395,17 \pm 0,62 b$ \\
\hline
\end{tabular}

Les valeurs moyennes de la même colonne indexée de la même lettre ne sont pas statistiquement différents selon le test de Turkey au seuil $5 \%$. VE : valeur énergétique.

Tableau 3: Ration de croissance.

\section{Composition chimique (\% MS)}

\begin{tabular}{|c|c|c|c|c|c|c|c|}
\hline & Matière Sèche & Humidité & Cendre & MatièresGrasses & Protéines & Glucides & VE (Kcal/100g) \\
\hline R0 & $93,5 \pm 0,01 \mathrm{a}$ & $6,50 \pm 0,01 \mathrm{a}$ & $6,18 \pm 0,08 a$ & $13,10 \pm 0,11 \mathrm{a}$ & $17,07 \pm 0,24 \mathrm{a}$ & $57,17 \pm 0,07 a$ & $414,82 \pm 0,25 a$ \\
\hline $\mathrm{R} 33$ & $93,62 \pm 0,01 \mathrm{a}$ & $6,40 \pm 0,02 \mathrm{a}$ & $6,28 \pm 0,04 a$ & $11,88 \pm 0,21 b$ & $22,79 \pm 0,27 b$ & $52,65 \pm 0,51 b$ & $408,71 \pm 0,84 b$ \\
\hline R66 & $93,66 \pm 0,02 \mathrm{a}$ & $6,37 \pm 0,03 a$ & $5,39 \pm 0,13 b$ & $9,11 \pm 0,25 c$ & $23,03 \pm 0,07 b$ & $56,09 \pm 0,06 \mathrm{c}$ & $398,51 \pm 0,40 c$ \\
\hline $\mathrm{R} 100$ & $93,77 \pm 0,03 \mathrm{a}$ & $6,28 \pm 0,04 a$ & $4,75 \pm 0,00 c$ & $7,70 \pm 0,11 d$ & $23,39 \pm 0,07 b$ & $57,89 \pm 0,16 \mathrm{~d}$ & $394,41 \pm 0,05 d$ \\
\hline
\end{tabular}

Les valeurs moyennes de la même colonne indexée de la même lettre ne sont pas statistiquement différents selon le test de Turkey au seuil 5\%. VE : valeur énergétique. 
Tableau 4: Gain Moyen Quotidien (GMQ).

\begin{tabular}{|c|c|c|c|c|c|}
\hline & GMQ (g/jour) & & & Traitements & \\
\hline & & Ro & $\mathbf{R 3 3}$ & R66 & R100 \\
\hline Démarrage & Semaine 3-5 & $42,98 \pm 18,69 a$ & $34,59 \pm 9,68 \mathrm{a}$ & $39,21 \pm 11,73 a$ & $40,26 \pm 16,19 a$ \\
\hline croissance & Semaine 6-7 & $56,48 \pm 3,17 b$ & $52,33 \pm 0,82 b$ & $56,14 \pm 6,79 b$ & $57,34 \pm 8,14 b$ \\
\hline Essai & semaine $3-7$ & $42,38 \pm 18,60 \mathrm{c}$ & $41,69 \pm 11,49 c$ & $45,99 \pm 13,03 c$ & $43,09 \pm 13,99 c$ \\
\hline
\end{tabular}

Les valeurs moyennes de la même ligne indexée de la même lettre ne sont pas statistiquement différents selon le test de Turkey au seuil $5 \%$.

Tableau 5: Consommation alimentaire individuelle (CAI) .

\begin{tabular}{|c|c|c|c|c|c|}
\hline & \multirow[t]{2}{*}{ CAI (g/sujet/j) } & \multicolumn{4}{|c|}{ Traitements } \\
\hline & & R0 & R33 & R66 & R100 \\
\hline Démarrage & Semaine 3-5 & $81,16 \pm 14,34 a$ & $74,48 \pm 5,83 a$ & $80,36 \pm 18,13 a$ & $79,92 \pm 15,50 a$ \\
\hline Croissance & Semaine 6-7 & $86,00 \pm 0,57 b$ & $70,59 \pm 0,00 b$ & $80,00 \pm 0,00 \mathrm{~b}$ & $86,00 \pm 0,00 b$ \\
\hline Essai & semaine $3-7$ & $87,10 \pm 13,28 \mathrm{c}$ & $72,92 \pm 4,90 \mathrm{c}$ & $80,22 \pm 14,05 c$ & $86,35 \pm 14,36 c$ \\
\hline
\end{tabular}

Les valeurs moyennes de la même ligne indexée de la même lettre ne sont pas statistiquement différents selon le test de Turkey au seuil $5 \%$.

Tableau 6: Indice de consommation (IC).

\begin{tabular}{|c|c|c|c|c|c|}
\hline \multicolumn{3}{|c|}{ I C } & \multicolumn{3}{|c|}{ Traitements } \\
\hline & & R0 & $\mathbf{R 3 3}$ & R66 & R100 \\
\hline Démarrage & Semaine $3-5$ & $2,32 \pm 1,00 \mathrm{a}$ & $2,29 \pm 0,49 a$ & $2,17 \pm 0,49 \mathrm{a}$ & $2,25 \pm 0,67 a$ \\
\hline Croissance & Semaine 6-7 & $1,45 \pm 0,06 b$ & $1,35 \pm 0,02 b$ & $1,45 \pm 0,17 b$ & $2,09 \pm 0,35 b$ \\
\hline Essai & semaine $3-7$ & $1,97 \pm 0,88 c$ & $1,91 \pm 0,60 \mathrm{c}$ & $1,88 \pm 0,53 c$ & $2,19 \pm 0,57 \mathrm{c}$ \\
\hline
\end{tabular}


Tableau 7: Rendements organes (carcasse, gras abdominal, cœur, foie et gésier).

\begin{tabular}{|c|c|c|c|c|}
\hline Rendement (\%) & R0 & R33 & R66 & R100 \\
\hline Rd carcasse & $80,02 \pm 3,18 \mathrm{a}$ & $92,62 \pm 5,51 b$ & $78,79 \pm 9,20 \mathrm{a}$ & $89,13 \pm 7,63 b$ \\
\hline Rd gras abdominal & $1,44 \pm 0,10 b$ & $1,86 \pm 0,18 b$ & $2,11 \pm 0,43 b$ & $2,00 \pm 0,68 b$ \\
\hline Cœur & $0,43 \pm 0,08 \mathrm{a}$ & $0,45 \pm 0,08 \mathrm{a}$ & $0,45 \pm 0,09 \mathrm{a}$ & $0,39 \pm 0,05 a$ \\
\hline Foie & $2,09 \pm 0,21 b$ & $3,09 \pm 0,19 \mathrm{c}$ & $2,38 \pm 0,27 b$ & $2,42 \pm 0,37 b$ \\
\hline Gésier & $1,93 \pm 0,16 \mathrm{~d}$ & $2,55 \pm 0,12 d$ & $2,18 \pm 0,79 d$ & $2,60 \pm 0,28 d$ \\
\hline
\end{tabular}

Tableau 8 : Mortalité des poulets de chair pendant la période d'essai.

\begin{tabular}{ccccccc}
\hline & Rations & R0 & R33 & R66 & R100 & Total \\
\hline & Effectif initial & 25 & 25 & 25 & 25 & 100 \\
\hline Démarrage & & \multicolumn{5}{c}{ Mortalités } \\
& semaine 3 & 0 & 0 & 0 & 0 & 0 \\
& semaine 4 & 0 & 1 & 0 & 0 & 1 \\
& semaine 5 & 0 & 3 & 2 & 0 & 5 \\
Croissance & semaine 6 & 0 & 4 & 8 & 0 & 12 \\
& semaine 7 & 0 & 0 & 0 & 0 & 0 \\
& Total & 0 & 8 & 10 & 0 & 18 \\
& Taux de Mortalité $(\%)$ & 0 & 32 & 40 & 0 & 18 \\
\hline
\end{tabular}




\section{DISCUSSION}

La farine de chenille constitue une importante source de protéine (53\%) pour l'alimentation humaine et animale. Les taux de protéine obtenus par la présente étude sont inférieurs à ceux (59-61\%) de plusieurs auteurs (Diomandé et al., 2008; Akpossan et al. 2009; Ouedraogo et al., 2015). Ces auteurs ont travaillé respectivement sur des farines d'escargot (Achatina fulica) comparées à celle de poisson dans l'alimentation de poulet de chair en Côte d'Ivoire et sur les farines d'asticots et co-produit de volailles dans l'alimentation de poulet au Burkina Faso. Les différentes techniques de transformation de ces farines et leur nature pourraient être les raisons de ces différences entre les teneurs en protéines. La farine de chenille présente une faible teneur en cendres ce qui implique que son incorporation dans les rations alimentaires de poulet doit s'accompagner de leur complémentation en minéraux. Sa teneur en matière grasse (19\%) est sensiblement identique à celle de poisson $(20 \%)$ dans cette étude. Cette teneur en matière grasse est supérieure à celle de poisson $(10 \%)$ et escargot (4\%) obtenue par Diomandé et al. (2008). Ce résultat indique que la farine de chenille peut être utilisée pour l'engraissement des poulets.

Les valeurs énergétiques et les teneurs en protéines des rations de cette étude sont plus élevées que celles de Abasse et al. (2017) et de Kana et al. (2015). Ces auteurs ont travaillé respectivement sur la farine de feuilles de Moringa oleifera et résidus de semoules de manioc supplémenté par la spiruline dans la production de poulets de chair. Cette différence pourrait être liée à la composition chimique des ingrédients utilisés dans les formulations des rations.

Les poids vifs des poulets ont augmenté durant l'essai de $300 \mathrm{~g}$ à $2241 \mathrm{~g}$ pour R0 et à $1785 \mathrm{~g}, 1908 \mathrm{~g}$ et $1803 \mathrm{~g}$ respectivement pour R33, R66 et R100. Cependant, l'analyse statistique n'a montré aucune différence significative entre les traitements $(p>0.05)$. Ces résultats sont supérieurs à ceux obtenus par plusieurs auteurs (Ouedraogo et al., 2015 (958,5 -
227,52 g); Gnikpo et al., 2016 (1100-1411 g). Abasse et et al. $2017(1000 \mathrm{~g}))$. Ces poids vifs des poulet de cette étude sont similaires à ceux de Kana et al., 2015 (1774-2266 g). Ces variations de poids vifs peuvent être liées à la nature, la composition chimique des ingrédients, les races des poulets et l'environnement des essais.

Les GMQ ont varié entre 41,6 et 52,3 $\mathrm{g} /$ jour. Ces valeurs sont supérieures à celles de Dongmo et al. (2000); Gnikpo et al. (2016) qui ont obtenu respectivement, au cours de leurs travaux, 19-27 g/jour et $20 \mathrm{~g} /$ jour. Ces résultats indiquent que la farine de chenille serait une excellente source de nutriments pour les volailles.

Les CAI variaient entre 72,9 et 87,1 $\mathrm{g} / \mathrm{sujet} / \mathrm{j}$. Ces valeurs sont plus élevées que celles de Dongmo et al. (2000) 51,8 à 68,3 $\mathrm{g} / \mathrm{sujet} / \mathrm{j}$. Ces résultats indiquent que les aliments supplémentés par les farines de chenille ont été plus appréciés et donc plus consommés par les poulets de chair.

Au cours de cet essai, les IC ont varié entre 1,8 et 2,1. Ces indices sont inférieurs à ceux obtenus par Dongmo et al., (2000), Diomandé et al. (2008) et Ouedraogo et al., (2015) qui ont obtenu respectivement 2,5 2,$4 ; 2,3-2,4$ et 2,3 - 2,7 pour des essais de farines animales dans les rations de poulet de chair. Les farines de chenille favoriseraient de meilleures conversions alimentaires au niveau des poulets de chair.

Les rendements carcasses (78-92\%) obtenus à l'issu de la présente étude sont similaires à ceux (64-94\%) obtenus par Diomandé et al. (2008) et supérieurs à ceux de Kana et al. (2015) (70,95-73,51\%). Quant aux rendements en gras abdominal des différents traitements, l'analyse statistique montre aucune différence significative $(p>0,05)$ entre ceux-ci. Les taux de gras abdominal obtenus dans la présente étude sont inférieurs à ceux de Diomandé et al. (2008). On en déduit que la farine de chenille favoriserait plus la formation de tissu musculaire au détriment des tissus adipeux (graisse) chez les sujets.

La similarité obtenue pour les poids du cœur et du gésier des sujets des traitements $\mathrm{R} 33$, R66 et R100 par rapport au témoin R0 
explique que la farine de chenille n'a eu d'effets néfastes marqués sur le fonctionnement de ces organes. En revanche, l'analyse statistique des données a montré une différence significative $(p<0,05)$ entre les poids $\mathrm{du}$ foie R33 et les autres. L'augmentation du poids du foie dans le traitement R33 peut être liée à la nécessité de cet organe d'augmenter son efficacité. En effet, le foie est un centre important de détoxification de l'organisme.

Les taux de mortalités sont notés pour les rations à farine de poisson et de chenille mélangée à $33 \%$ et $66 \%$ d'incorporation de farine de chenille. Lorsque les rations sont à $100 \%$ de farine de poisson ou de farine de chenille il n'y a pas de mortalités .Le mélange de farine de chenille à la farine de poisson influencerait la mortalité. Ces résultats sont en accord avec ceux de Ouedraogo et al. (2015) qui ont montré que les mortalités sont élevées pour les régimes base d'asticots. En effet, un régime trop riche en protéines animale induirait une augmentation de la calciurie qui est un facteur de risque de la lithiase calcique ou calculs rénaux.

\section{Conclusion}

$\mathrm{Au}$ terme de l'expérimentation, il ressort que la farine de chenille est plus riche en protéines $(53,57 \%)$ que celle de poisson $(38,56 \%)$. Elle est plus pauvre en cendres $(2,17 \%)$ que la farine de poisson $(7,73 \%)$.

L'évaluation des performances zootechniques des poulets nourris aux 4 rations a montré des variations non significatives des poids vifs (1803-2241 g), gains moyens quotidiens $(41,62-45,99$ $\mathrm{g} /$ sujet), consommations alimentaires individuelles $(72,92-87,10 \mathrm{~g} / \mathrm{sujet} / \mathrm{j})$, indices de consommation $(1,88-2,19)$ selon les taux variables de farine de chenilles dans les rations des sujets. Il en a été de même que les rendements carcasses $(78,79-92,62 \%)$, gras abdominaux (1,44-2,11\%) cœur $(0,39$ $0,45 \%)$, foie $(2,09-3,09 \%)$, gésier $(1,93-$ $2,60 \%)$. La mortalité n'a pas été corrélée avec les teneurs de farine de chenille des régimes alimentaires des poulets.
Ainsi, la chenille (Imbrasia oyemensis) peut être valablement incorporée dans l'alimentation des poulets de chair sans risque de modifier la production et les caractéristiques des viandes. Elle peut être recommandée dès lors aux aviculteurs comme ressource alternative de protéines pour la volaille dans les pays tropicaux.

Les déterminations des acides aminés, des acides gras et des facteurs antinutritionnels de la farine de chenille (Imbrasia oyemensis) sont nécessaires pour mieux valoriser cette chenille en nutrition animale et humaine.

\section{CONFLIT D'INTERETS}

Les auteurs déclarent qu'ils n'ont pas de conflit d'intérêts.

\section{CONTRIBUTIONS DES AUTEURS}

MD a assuré la conception du protocole, la conduite de l'expérience et la rédaction du manuscrit. MGAB a contribué à la rédaction du manuscrit. BKK a assuré le traitement statistique des données. LGB a contribué à la conception du protocole expérimental.

\section{RÉFÉRENCES}

Abasse T, Maigachi I, Habba W, Diallo D, 2017. Effet de la supplementation de la farine des feuilles de moringa oleifera (Lam) dans la production des poulets de chair au Niger. Int. J. Biol. Chem. Sci., 11(2): 722-729. DOI: https://dx.doi.org/104314/ijbcs.v11i2.17

Akpossan RA, Ahipo DE, Kouadio JPEN, Kouame LP. 2009- Nutritional and physicochemical characterization of the fat caterpillar (Imbrasia oyemensis) dried and sold at market Adjame (Abidjan, Ivory coast). J. Anim. plant Sci., 3(3): 243-250.

AOAC. 1995. Official Methods of Analysis of $A O A C$ International $\left(16^{\text {th }}\right.$ edn). AOAC International Arlington, VA; 250.

Atwater WO, Benedict FG. 1902. Experiments on the Metabolism of Matter and Energy in the Human Body, 1898-1900. Office of experiment 
stations. Bulletin No. 109. Government Printing Office: Washington, DC, United States.

Bertrand G, Thomas P. 1910. Guide pour les Manipulations de Chimie Biologie. Dund : Paris

BIPEA. 1976. Recueil des Méthodes d'Analyse des Communautés Européennes. Bureau Interprofessionnel d'Études Analytiques, Gennevilliers. France ; 140.

Diomandé M, Koussemon M, Allou KV, Kamenan A. 2008. Effect of snail (Achatina fulica) meal broiler production and meat sensorial quality, Livestock Research for Rural Developpement, 20 (12) paper 2.

Dongmo T, Ngou NJD, Pouille DMJ. 2000. Utilisation de quelques farines animales locales dans l'alimentation du poulet de chair, Tropicultura, 18(3): 122-125.

Doumbia F. 2002. L'approvisionnement en intrants de la filière avicole moderne au Sénégal. Thèse, Méd. Vét : Dakar ; 27p.

Essoh, AF. 2006. Les importations de viandes de volaille et la filière avicole en Côte d'Ivoire de 1999 à 2003. Thèse de Doctorat de l'École Inter-états des sciences et Médecine Vétérinaires (E.I.S.M.V) de Dakar (Sénégal), 153p.

Gnipko AF, Chrystome CAAM, Houndonougbob FM, Adenile AD, Dougnon TJ, Codjia JTC. 2016. Effectiveness of probiotic feed ingredient on the growth performance of broiler. Int. J. Biol. Chem. Sci., 10(3):
1163-1172. DOI: https:

//dx.doi.org/104314/ijbcs.v10 i3.21

Kana JR, Hassan AD, Ngouana TR, Mube KH, Teguia A, TefackY, Zambou HR. 2015. Performances zootechniques de poulet de chair soumis à un régimeà base de résidus de soumoule des maniocs supplementé par la spiruline (Spirulina platensis). Int. J. Biol. Chem. Sci., 9(5): 2607-2616.

DOI: https://dx.doi.org/10.4314/ijbcs.v9 i5.30

Malaisse F, Lognay G. 2004. Les chenilles comestibles d'Afrique tropicale. In Les Insectes dans Latradition Orale, MotteFlorac E, Thomas JMC (eds) Ethnoscience 5, Peeters, Selaf $\mathrm{n}^{\circ} 407$, Ethnosciences 11 : Paris ; 279-304.

Ouedraogo B, Gnanda IB, SANFO R, Zoundi SI, Bayala B. 2015. Etude comparative des performances réalisées avec l'incorporation de la farine de coproduits de volaille et la farine des asticots dans des rations de poulets de chair au Burkina faso. Rev. Ivoir.Sci. Technol., 25, 148-161.

Pousga SH, Boly B, Ogle A. 2005. Choice feeding of poultry: a review. Livestock Research for Rural Development, 17(4).

Sonaiya BE, Gueye ELHF. 1998. Bulletin RIDAF (3) Thése de Méd. Vét, Dakar, $78 \mathrm{p}$. 